\title{
Article
}

\section{Predictive Value of Measures of Vascular Calcification Burden and Progression for Risk of Death in Incident to Dialysis Patients}

\author{
Antonio Bellasi ${ }^{1, *,+} \oplus$, Luca Di Lullo ${ }^{2}$, Domenico Russo ${ }^{3}$, Roberto Ciarcia ${ }^{4} \oplus^{\circ}$, Michele Magnocavallo ${ }^{5}(\mathbb{D}$,

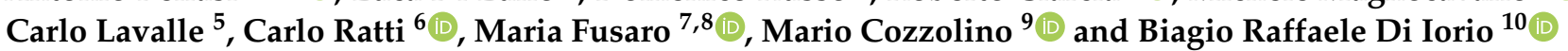

1 Department of Research, Innovation and Brand Reputation, ASST Papa Giovanni XXIII, 24127 Bergamo, Italy

2 Department of Nephrology and Dialysis, Ospedale Parodi, Delfino, 00034 Colleferro, Italy; dilulloluca69@gmail.com

3 Department of Nephrology, School of Medicine, University “Federico II”, 80125 Napoli, Italy; domenicorusso51@hotmail.com

4 Departments of Vetecerinary Medicine and Animal Productions, University of Naples Federico II, 80137 Naples, Italy; roberto.ciarcia@unina.it

5 Department of Clinical, Internal, Anesthesiology and Cardiovascular Sciences, Policlinico Universitario Umberto I, Sapienza University of Rome, 00185 Roma, Italy; michelefg91@gmail.com (M.M.); carlolavalle@yahoo.it (C.L.)

6 Department of Cardiology, Ospedale Ramazzini, 41012 Carpi, Italy; ratticarlo@hotmail.com

7 National Research Council (CNR)-Institute of Clinical Physiology (IFC), 56124 Pisa, Italy; dante.lucia11@gmail.com

8 Department of Medicine, University of Padua, 35122 Padua, Italy

9 Renal Division, Department of Health Sciences, ASST Santi Paolo e Carlo, University of Milan, 20142 Milan, Italy; mario.cozzolino@unimi.it

check for

updates

Citation: Bellasi, A.; Di Lullo, L.; Russo, D.; Ciarcia, R.; Magnocavallo, M.; Lavalle, C.; Ratti, C.; Fusaro, M.; Cozzolino, M.; Di Iorio, B.R. Predictive Value of Measures of Vascular Calcification Burden and Progression for Risk of Death in Incident to Dialysis Patients. J. Clin. Med. 2021, 10, 376. https://doi.org/10.3390/jcm10030376

Received: 22 December 2020

Accepted: 17 January 2021

Published: 20 January 2021

Publisher's Note: MDPI stays neutral with regard to jurisdictional claims in published maps and institutional affiliations.

Copyright: (c) 2021 by the authors. Licensee MDPI, Basel, Switzerland. This article is an open access article distributed under the terms and conditions of the Creative Commons Attribution (CC BY) license (https:// creativecommons.org/licenses/by/ $4.0 /)$.
10 Nefrology and Dialysis, AORN “San Giuseppe Moscati”, 83100 Avellino, Italy; br.diiorio@gmail.com

* Correspondence: abellasi@asst-pg23.it

+ Current address: Department of Research, Innovation and Brand Reputation, ASST Papa Giovanni XXIII, P.za OMS 1, 24127 Bergamo, Italy.

Abstract: Background: Vascular calcification (VC) is a marker of cardiovascular (CV) disease and various methods allow for presence and extension assessment in different arterial districts. Nevertheless, it is currently unclear which one of these methods for VC evaluation best predict outcome and if this piece of information adds to the predictive value of traditional CV risk factors in patients receiving hemodialysis (HD). Methods: data of 184 of the 466 patients followed in the Independent study (NCT00710788) were post hoc examined to assess the association three concurrent measures of vascular calcification and all-cause survival. Specifically, coronary artery calcification (CAC) was determined by the Agatston and the volume score while abdominal aorta calcification was determined by plain X-ray of the lumbar spine (Kauppila score (KS)). Survival and regression models as well as metrics of risk recalculation were used to test the association of VC and outcome beyond the Framingham risk score. Results: Middle-age (62.6(15.8) years) men (51\%) and women (49\%) starting HD were analyzed. Over 36 (median 36; interquartile range: 8-36) months of follow-up 69 patients expired. Each measure of VC (CAC or KS) predicted all-cause mortality independently factors commonly associated with all-cause survival $(p<0.001)$. Far more importantly, each measurement of VC significantly improved risk prediction and patient reclassification $(p<0.001)$ beyond traditional cardiovascular risk factors. Conclusions: Overall, presence and extension of VC, irrespective of the arterial site, predict risk of all-cause of death in patients starting hemodialysis. Of note, both CAC and KS increase risk stratification beyond traditional CV risk factors. However, future efforts are needed to assess whether a risk-based approach encompassing VC screening to guide HD patient management improves survival.

Keywords: vascular calcification; coronary artery calcification; aorta calcification; hemodialysis; risk prediction 


\section{Introduction}

Vascular calcification (VC) is a useful marker of cardiovascular disease and several methods are available for the assessment of their presence and extension [1-5]. Although the pathogenesis of VC is not well established, several studies suggest that the prevalence of VC increases as renal function declines, likely due to the many metabolic abnormalities that characterize chronic kidney disease (CKD) $[3,5,6]$. Irrespective of the noxious mechanism responsible for VC deposition and progression, the data suggest that the risk of unfavorable outcome is higher for greater VC burden [2,5]. As alluded by the Kidney Disease Improving Global Outcome (KDIGO) clinical guideline for Chronic Kidney Disease and Mineral Metabolism Disease (CKD-MBD) management, VC, as a marker of vascular disease, may allow for risk prediction refinement as well as for individualized patients management [7].

Although coronary artery calcification (CAC) has been traditionally used to detected presence and extension of vascular calcification, several other less expensive and widely available tools are available to assess VC in different arterial sites as well as vascular risk [2,5]. In these regards, the Kauppila score (KS) using lateral-lateral plain X-ray of the lumbar spine has been proposed to evaluate $\mathrm{VC}$ in the abdominal aorta. A few reports suggest a close correlation of KS and CAC as well as KS and risk of death in dialysis patients [1-5]. However, which VC measures best predicts long-term survival and whether a measure of vascular calcification adds to the predictive value of traditional Framingham risk stratification in incident to hemodialysis (HD) patients, has not been determined through a concurrent comparison of these measures in a single prospective cohort [5].

For the present study we examined the association of CAC evaluated by 2 different scores systems namely the Agatston score [8] and the volume score [9], as well as Abdominal Aorta Calcification (AAC) evaluated via lateral-lateral plain X-ray of the lumbar spine (KS) [10] and the risk of all-cause mortality. Metrix of risk prediction reclassification according to presence end extension of VC are also investigated for each of the three measures of VC.

\section{Material and Methods}

\subsection{Study Cohort and Endpoint of Interest}

We utilized data from patients incident to hemodialysis recruited in the INDEPENDENT study (ClinicalTrials.gov: NCT00710788) [11,12]. Briefly, the Independent study was designed to assess the impact of 2 different phosphate binder regimens (calcium free vs. calcium containing-phosphate binder) on Cardiovascular (CV) events as well as all-cause mortality [11,12]. In the Independent study 466 adult patients ( $>18$ years) new to hemodialysis (requiring dialysis $<120$ days) were randomized in a 1:1 fashion at 18 dialysis center in Italy to receive open-label sevelamer or calcium carbonate/calcium acetate as phosphate binder [11,12]. Age older than 75 years, history of cardiac arrhythmia, syndrome of congenital prolongation of the QT segment interval, a corrected QT (QTc) longer than $440 \mathrm{~ms}$ or increased QT dispersion (QTd), history of coronary artery bypass (CABG), liver dysfunction and hypothyroidism and use of drugs that prolong the QT interval were regarded as exclusion criteria [11,12]. Enrollment began in September 2006 and continued through July 2008 [11,12]. Study follow-up ended in July 2011 [11,12]. Written informed consent was obtained from all participants prior to study entry and after approval from each institutional Ethical Review Board. The study was conducted in adherence to the Declaration of Helsinki, Ethical Principles for Medical Research Involving Human Subjects [11,12].

As part of the study protocol, CAC was evaluated at baseline and after 12 months from study inception [11,12]. Furthermore, in a subgroup $(n=184,39 \%$ of the Independent study cohort) of patients AAC was evaluated at study inception according to the method described by Kauppila and coworkers. Of importance, this last evaluation was not mandate by the study protocol and left at the physician discretion.

The endpoint of interest was defined as all-cause of mortality and, by study design, all patients were followed until death or study completion (36 months of follow-up) [11,12]. 
During follow-up, the investigators were instructed to control blood pressure (blood pressure target: below 130/80 mm Hg), anemia (Hb below $11 \mathrm{~g} / \mathrm{dL}$, TSAT below 20\%), acidosis (HCO3 between 20 and $24 \mathrm{mmol} / \mathrm{L})$, diabetes $(\mathrm{HbA} 1 \mathrm{c}<7.0 \%$ ), dyslipidemia (total cholesterol below $200 \mathrm{mg} / \mathrm{dL}$; LDL cholesterol below $100 \mathrm{mg} / \mathrm{dL}$; triglycerides below $180 \mathrm{mg} / \mathrm{dL}$ ), and the parameters of bone mineral metabolism (serum phosphorous 2.5-5.0 mg/dL, serum calcium 8.0-9.9 mg/dL, and intact-PTH between 150-300 pg/mL) according to guidelines available at the time the study was conceived $[11,12]$.

\subsection{Demographic, Clinical and Laboratory Characteristics as Well as Vascular Calcification Assessment}

Demographic, clinical and laboratory characteristics were collected at the Independent study initiation [11,12]. Risk of atherosclerotic event were assessed via the Framingham risk score, as previously described [13]. History of atherosclerotic disease (ASCVD) was defined if any of the following clinical data was reported: history of cerebrovascular disease; peripheral vascular disease; angina pectoris; history of myocardial infarction; aortic aneurysm; history of percutaneous coronary angioplasty with or without stenting.

Routine biochemical laboratory measurements were obtained at baseline and at 6-monthly intervals. For current analysis only data on baseline measurements are considered. All blood samples were taken before the midweek dialysis session and after $12 \mathrm{~h}$ fasting. Serum parameters of mineral metabolism, electrolytes, anemia and dialysis adequacy were performed by the usual laboratories of the facilities [11,12].

Vascular calcification and arterial stiffness were evaluated at study entry and at 6-monthly intervals for the first 24 months of study follow-up [11,12]. CAC was assessed by a multislice lightspeed (GE Medical Systems) equipment at one center (Solofra, Italy). A standard imaging protocol was used to acquire a set of ECG-gated tomographic slices from the carina to the diaphragm. For each area of interest identified along the course of the coronary arteries was calculated as originally described by Agatston et al. (Agatston score) [8] as well as Callister et al. (volume score) [9]. To reduce inter-reader variability, all CAC scores were obtained in a single central location. No scan inter- as well as intra-reader variability assessment was performed in the Independent study. However, the reported variability for the Agatston and volume score is about $8-10 \%$ [1].

AAC was evaluated by lateral-lateral plain roentgenography via a technique similar to that described by Kauppila et al [10]. In brief, a lateral plain radiograph of the abdomen incorporating the first 4 lumbar vertebrae (L1 to L4) was obtained. The aorta was identified as the tubular structure coursing in front of the anterior surface of the spine and VC scored according to the length of each calcified plaque identified along the anterior and posterior profile of the aorta (points were assigned from 1 to 3: 1; small; 2 moderate; 3: large). With this numerical grading, the score could vary from a minimum of 0 to a maximum of 24 points (Kauppila score (KS)) [11]. Due to the fact that KS was not protocolized, lateral-lateral plain X-rays of the lumbar spine were performed at each participating center and read by the attending physician. Inter- and intra- reader agreement for the KS ranges between 0.71-0.85 and 0.93-0.95, respectively [11].

Arterial stiffness was assessed through carotid-femoral Pulse Wave Velocity (PWV) measurement. PWV was evaluated by applanation tonometry with Pulse Pen (Diatecne, Milan, Italy) as previously described as the ratio between the distance (meter) and the travelling time (second) of the pulse generated each cardiac cycle. Hence, PWV is expressed as m/s and higher value represent stiffer arteries [14].

\section{Statistical Analysis}

In this post-hoc analysis, no adjustment for multiple comparisons was made. Data are expressed as mean (Standard deviation-SD) or median [interquartile range] when appropriate. Categorical variables are presented as number of patients (\%). Parametric and non-parametric tests were used to compare demographic and clinical characteristics according to the occurrence of any lethal event before study completion (36 months follow-up). Owing to the skewness of the CAC score, baseline CAC was log transformed $[\log (\mathrm{CAC}+1)]$ 
if used as continuous variable or categorized as CAC $=0$, CAC between 1-99, CAC between 100-399 or CAC $=400+$ if used as categorical variable [15]. The semi-quantitative Kauppila score is represented as continuous variable or quartiles of the study distribution when appropriate. Comparison between patients with and without AAC evaluation are presented in the Table S1.

To gauge the association between different measures of vascular calcification (i.e., CAC evaluated via Agatston as well as Volume score and AAC) and all-cause mortality, survival analyses were used. Cumulative mortality curves were calculated by VC burden category using the Kaplan-Meier product-limit method while the hazard of all-cause death associated with VC burden as continuous variable was estimated using Cox survival analyses. A multi-step approach was carried out. First, we identified independent predictors of all-cause mortality in the study cohort. All variables associated with death at univariate analyses (variable with $p<0.15$ ) and based on available evidence were forced into a multivariable adjusted Cox-model (Table S2A). Then, a stepwise approach was employed to identify the most parsimonious model to predict the outcome of interest (Table S2B). Subsequently, the independent association of each individual measurement of VC and all-cause mortality was tested against the independent predictors of death (fully adjusted model: adjusted for measure of VC, Pulse Wave Velocity, age, Framingham score, diabetes, ASCVD, systolic blood pressure, serum levels of phosphate, calcium, PTH, use of ARBs, beta-blockers, vitamin D, calcium containing phosphate binder, calcium channel blockers and cinacalcet). Consistently with the methodology used to identify predictors of all-cause mortality, the most parsimonious model to predict death was identified through a stepwise procedure starting from the fully adjusted model.

Logistic regression was utilized to evaluate the incremental ability of each measurement of VC to predict all-cause mortality by computing the C-index, calibration statistic, integrated discrimination improvement (IDI) and continuous net reclassification improvement (NRI) of the most parsimonious model reported in with or without measurement of VC). Further, the discriminative value of the model with and without the measurement of VC was also investigated by means of receiver operating characteristic (ROC).

Statistical significance was set at 0.05 . All analyses were completed using $\mathrm{R}$ version 3.6.2 (21 December 2019; The R Foundation for Statistical Computing, Vienna, Austria).

\section{Results}

Overall, we investigated 184 middle age subjects (39\% of the study cohort of the Independent study) with measurement of CAC and AAC at study inception available. The main characteristics of the study cohort are reported in Table 1. Because AAC assessment was not mandatory and left at the physician discretion some differences between subject recruited in the Independent study with and without AAC assessment are present (Table S1).

Table 1. Patients Characteristics according to study cohort and status at study completion.

\begin{tabular}{|c|c|c|c|c|}
\hline & Total $(n=184)$ & Alive $(n=115)$ & Expired $(n=69)$ & \\
\hline Variable & Mean (SD) or $n(\%)$ & Mean (SD) or $n(\%)$ & Mean (SD) or $n(\%)$ & $p$-Value \\
\hline \multicolumn{5}{|c|}{ Demographic and Clinical Characteristics } \\
\hline Age (years) & $62.6(15.8)$ & $58.5(15.1)$ & $69.4(14.5)$ & $<0.0001$ \\
\hline Male (\%) & $94(51.0 \%)$ & $57(49.5 \%)$ & $37(53.6 \%)$ & 0.703 \\
\hline Body Weight (Kg) & $68.3(13.1)$ & $69.2(13.5)$ & $66.7(12.4)$ & 0.198 \\
\hline $\operatorname{ASCVD}(\%)$ & $27(14.6 \%)$ & $14(12.1 \%)$ & $13(18.8 \%)$ & 0.307 \\
\hline Diabetes (\%) & $40(21.7 \%)$ & $22(19.1 \%)$ & $18(26.0 \%)$ & 0.356 \\
\hline Systolic Blood Pressure (mmHg) & $135(18)$ & $132(18)$ & $139(17)$ & 0.012 \\
\hline Diastolic Blood Pressure (mmHg) & $75(9)$ & $75(9)$ & $75(10)$ & 0.978 \\
\hline Framingham score (unit) & $11.8(3.5)$ & $11.1(3.7)$ & $13.0(2.9)$ & $<0.001$ \\
\hline
\end{tabular}


Table 1. Cont.

\begin{tabular}{|c|c|c|c|c|}
\hline & Total $(n=184)$ & Alive $(n=115)$ & Expired $(n=69)$ & \\
\hline Variable & Mean (SD) or $n(\%)$ & Mean (SD) or $n(\%)$ & Mean (SD) or $n(\%)$ & $p$-Value \\
\hline \multicolumn{5}{|c|}{ Measurement of Vascular Calcification \& Arterial Stiffness } \\
\hline CAC Agatston score & $569(1098)$ & $226(579)$ & $1139(1468)$ & $<0.0001$ \\
\hline CAC Agatston score progression & - & - & - & \\
\hline CAC Volume score & $229(334)$ & $112(223)$ & $423(393)$ & $<0.0001$ \\
\hline Abdominal Aorta VC (Kauppila score) & $13(9)$ & $10(8)$ & $18(7)$ & $<0.0001$ \\
\hline Pulse Wave Velocity $(\mathrm{m} / \mathrm{s})$ & $9.5(3.7)$ & $9.2(3.7)$ & $10.0(3.8)$ & 0.156 \\
\hline \multicolumn{5}{|c|}{ Laboratory Characteristics } \\
\hline Albumin (g/dL) & $3.7(0.4)$ & $3.7(0.3)$ & $3.7(0.4)$ & 0.794 \\
\hline Creatinine $(\mathrm{g} / \mathrm{dL})$ & $7.9(2.5)$ & $8.0(2.4)$ & $7.6(2.8)$ & 0.376 \\
\hline Hemoglobin $(\mathrm{g} / \mathrm{dL})$ & $11.0(1.6)$ & $11.1(1.7)$ & $10.9(1.3)$ & 0.428 \\
\hline Sodium (mE/L) & $139(3.5)$ & $139(3.7)$ & $139(2.9)$ & 0.152 \\
\hline Potassium (mEq/L) & $5.1(0.7)$ & $5.0(0.7)$ & $5.2(0.7)$ & 0.175 \\
\hline Calcium (mg/dL) & $8.8(0.9)$ & $8.9(0.9)$ & $8.6(0.7)$ & 0.036 \\
\hline Phosphate (mg/dL) & $4.5(1.3)$ & $4.4(1.2)$ & $4.8(1.4)$ & 0.055 \\
\hline Parathyroid Hormone (pg/mL) & $259(227)$ & $236(180)$ & $298(287)$ & 0.111 \\
\hline C-reactive protein $(\mathrm{mg} / \mathrm{L})$ & $5.0(3.3)$ & $4.9(3.6)$ & $5.1(2.8)$ & 0.762 \\
\hline \multicolumn{5}{|c|}{ Concomitant Medications } \\
\hline Use of ACE-inhibitors (\%) & $132(71.7 \%)$ & $78(67.8 \%)$ & $54(78.2 \%)$ & 0.176 \\
\hline Use of ARBs (\%) & $148(80.4 \%)$ & $87(75.6 \%)$ & $61(88.4 \%)$ & 0.055 \\
\hline Use of betablockers (\%) & $115(62.5 \%)$ & $81(70.4 \%)$ & $34(49.2 \%)$ & 0.007 \\
\hline Use of calcium channel blockers (\%) & $56(30.4 \%)$ & $27(23.4 \%)$ & $29(42.0 \%)$ & 0.013 \\
\hline Use of cinacalcet $(\%)$ & $79(42.9 \%)$ & $44(38.2 \%)$ & $35(50.7 \%)$ & 0.134 \\
\hline Use of vitamin D (\%) & $111(60.3 \%)$ & $78(67.8 \%)$ & $33(47.8 \%)$ & 0.011 \\
\hline Use of Sevelamer (\%) & $29(15.7 \%)$ & $23(20.0 \%)$ & $6(8.7 \%)$ & 0.067 \\
\hline Use of calcium based binders (\%) & $155(84.2 \%)$ & $92(80.0 \%)$ & $63(91.3)$ & 0.067 \\
\hline
\end{tabular}

ASCVD: atherosclerotic cardiovascular disease defined if any of the following clinical data was reported: history of cerebrovascular disease; peripheral vascular disease; angina pectoris; history of myocardial infarction; aortic aneurysm; history of percutaneous coronary angioplasty with or without stenting; CAC: coronary artery calcification; VC: vascular calcification; ARB: angiotensin receptor blocker.

Over a median follow-up of 36 [interquartile range IQR: 8-36] months, 69 patients expired. Patients who died of all-cause during follow-up were older [69(14) vs. 58(15)], exhibited higher systolic blood pressure [139(17) mmHg vs. 132(18) mmHg], Framingham risk score [13.0(2.9) vs. 11.1(3.7)], coronary as well as aortic aorta calcium burden and arterial stiffness (Table 1). Furthermore, differences in CKD-MBD as well as treatments were also apparent (Table 1).

To gauge the independent contribution of all factors associated with mortality, all variables significantly associated with death at univariate analyses (Table $1 \mathrm{~A}$, variable with $p<0.15$ ) were forced into a multivariable adjusted Cox-model (Table S2A) and the most parsimonious model was selected via a stepwise method (Table S2B). Accordingly, increased CAC burden and pulse wave velocity, history of diabetes mellitus and use of calcium containing phosphate binders were among the others the most relevant factors associated with risk of death (Table S2B).

Notably, at unadjusted analysis all 3 measurements (CAC assessed via the Agatston and volume score as well as AAC) were significantly associated $(p<0.001)$ with the risk of all-cause mortality Figure $1 \mathrm{~A}-\mathrm{C}$. 
Nonetheless, the independent association of CAC or KS was tested against the predictors of death Table 2A-C. In general, measures of CAC either assessed via the Agatston or the volume score systems and AAC were independently and strongly related with risk of death irrespectively of the baseline atherosclerotic risk assessed via the Framingham score or history of ASCVD (Table 2A-C). Furthermore, addition of VC increased the overall performance of each model (significant AIC increase with addition of measurement of VC to the model) Table 2A-C.

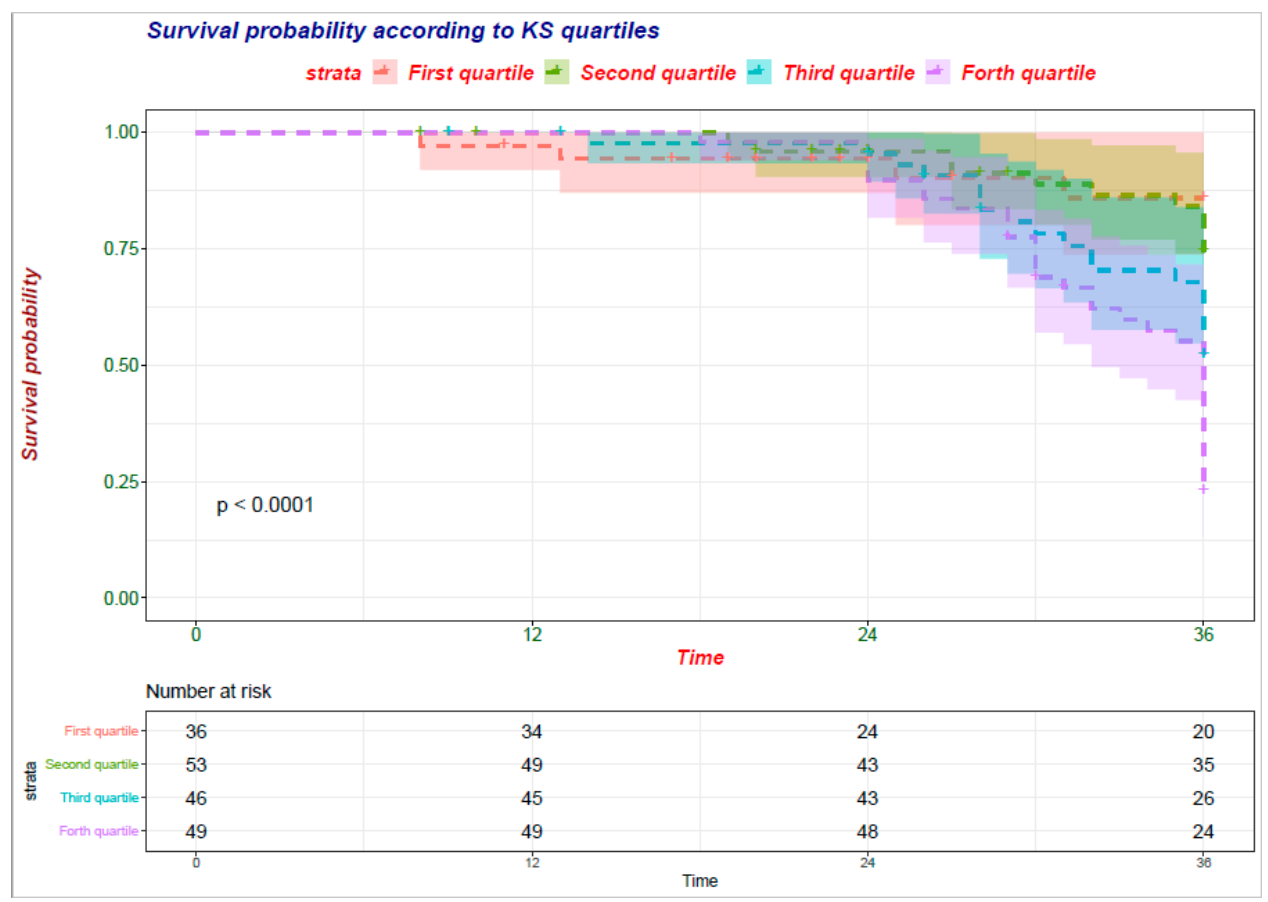

(A)

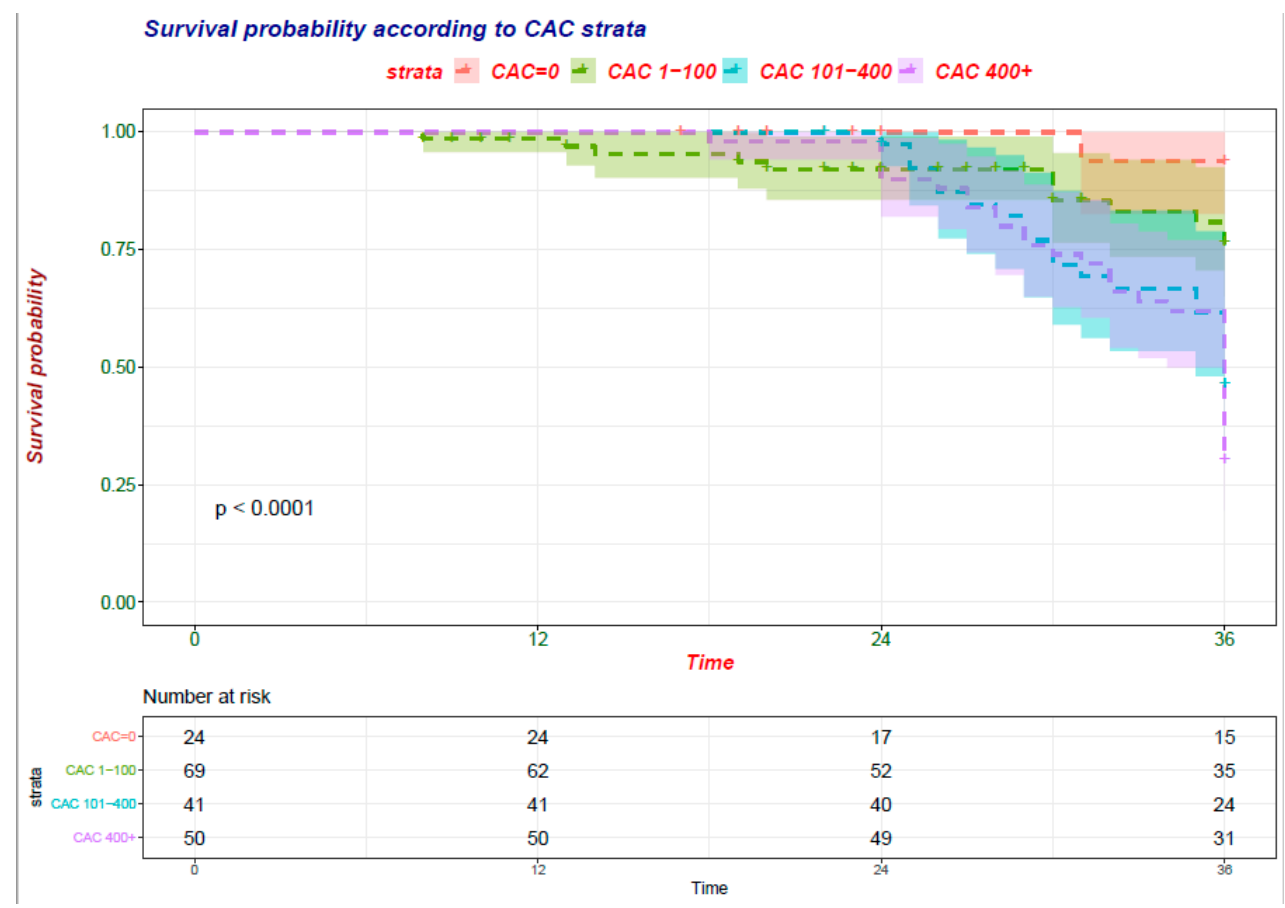

(B)

Figure 1. Cont. 


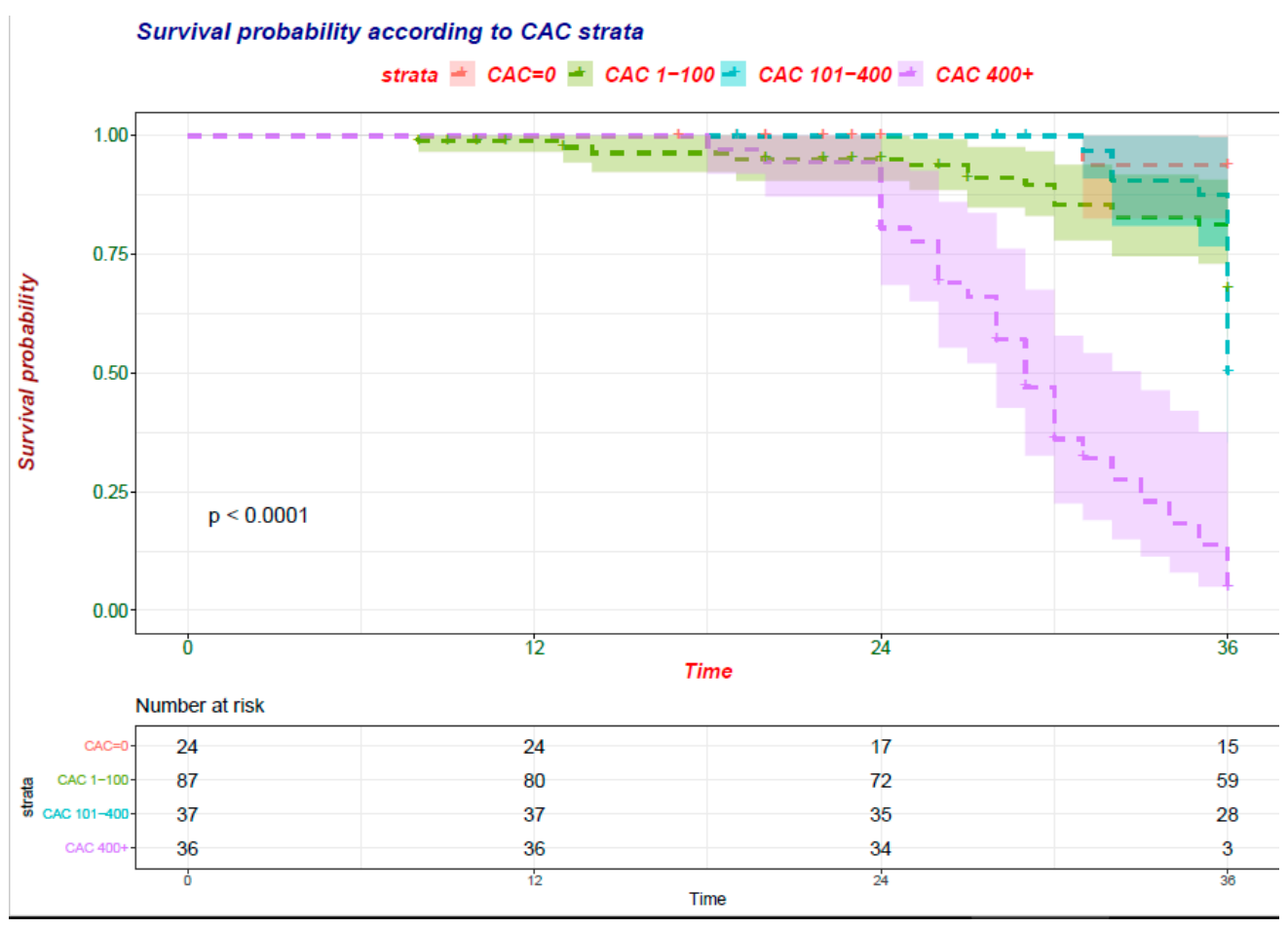

(C)

Figure 1. Cumulative mortality curves were calculated by VC burden category according to the Kaplan-Meier product-limit method. (A) Abdominal Aorta Calcification (AAC) evaluated by quartiles of Kauppila score (KS); (B) Coronary Artery Calcification (CAC) evaluated via the Agatston score; (C) CAC evaluated via the volume score.

Table 2. Independent predictors of all-cause mortality in the study cohort 1 according to measurement of Vascular calcification. (A) Abdominal Aorta Calcification (AAC) evaluated via the Kauppila score; (B) Coronary Artery Calcification (CAC) evaluated via the Agatston score; (C) CAC evaluated via the volume score. For each measurement of VC, the most parsimonious survival Cox models selected via a stepwise approach is presented. The fully adjusted model included predictors of all-cause mortality identified (reported in Table S2B).

(A) Abdominal Aorta Calcification (AAC) Evaluated via the Kauppila Score

\begin{tabular}{|c|c|c|c|c|}
\hline Variable & HR & Lower 0.95 & Upper 0.95 & $\operatorname{Pr}(>|z|)$ \\
\hline Kauppila score (1U increase) & 1.095 & 1.0577 & 1.133 & $<0.001$ \\
\hline Pulse wave velocity $(\mathrm{m} / \mathrm{s})$ & 1.061 & 0.9961 & 1.13 & 0.0658 \\
\hline Age (years) & 1.019 & 1.0002 & 1.038 & 0.0473 \\
\hline Systolic blood pressure (mmHg) & 1.013 & 0.9987 & 1.027 & 0.0747 \\
\hline Use of calcium channel blockers (y vs. $n$ ) & 1.476 & 0.8923 & 2.44 & 0.1295 \\
\hline Model fit Statistics (AIC-adding Kauppila) & \multicolumn{4}{|c|}{624.31 (final model with Kauppila) } \\
\hline loglink (without Kauppila) & \multicolumn{4}{|c|}{332.34} \\
\hline logling (with Kauppila) & \multicolumn{4}{|c|}{316.34} \\
\hline Comparison with vs. without Kauppila score & \multicolumn{4}{|c|}{ Chisq $31.89(p<0.001)$} \\
\hline
\end{tabular}


Table 2. Cont.

\begin{tabular}{|c|c|c|c|c|}
\hline \multicolumn{5}{|c|}{ (B) Coronary Artery Calcification (CAC) Evaluated via the Agatston Score } \\
\hline Variable & HR & Lower 0.95 & Upper 0.95 & $\operatorname{Pr}(>|z|)$ \\
\hline CAC-Agatstone score (log increase) & 1.6279 & 1.4176 & 1.869 & $<0.001$ \\
\hline Pulse wave velocity (m/s) & 1.1023 & 1.011 & 1.202 & 0.0273 \\
\hline Diabetes (y vs. $n$ ) & 3.597 & 1.7437 & 7.42 & 0.00053 \\
\hline ASCVD (y vs. n) & 0.5582 & 0.2718 & 1.146 & 0.11226 \\
\hline Systolic blood pressure (mmHg) & 1.011 & 0.9974 & 1.025 & 0.11198 \\
\hline Use of calcium containing phosphate binder (y vs. $n$ ) & 2.9523 & 0.9032 & 9.65 & 0.07321 \\
\hline Use of calcium channel blockers (y vs. $\mathrm{n}$ ) & 1.9427 & 1.1263 & 3.351 & 0.01696 \\
\hline Model fit statistics (AIC-adding CAC) & \multicolumn{4}{|c|}{624.31 (final model with CAC) } \\
\hline loglink (without CAC) & \multicolumn{4}{|c|}{305.16} \\
\hline logling (with CAC) & \multicolumn{4}{|c|}{333.41} \\
\hline Comparison with vs. without CAC score & \multicolumn{4}{|c|}{ Chisq $43.697(p<0.001)$} \\
\hline \multicolumn{5}{|c|}{ (C) Coronary Artery Calcification (CAC) Evaluated via the Volume Score } \\
\hline Variable & HR & Lower 0.95 & Upper 0.95 & $\operatorname{Pr}(>|z|)$ \\
\hline CAC-Volume score (log increase) & 1.7301 & 1.4469 & 2.069 & $<0.001$ \\
\hline Pulse wave velocity (m/s) & 1.0968 & 1.0082 & 1.193 & 0.03158 \\
\hline Age (years) & 1.0167 & 0.9967 & 1.037 & 0.10152 \\
\hline Diabetes (y vs. n) & 3.1042 & 1.4553 & 6.622 & 0.00338 \\
\hline ASCVD (y vs. n) & 0.5692 & 0.282 & 1.149 & 0.11584 \\
\hline Systolic blood pressure (mmHg) & 1.0103 & 0.9966 & 1.024 & 0.13968 \\
\hline Use of calcium containing phosphate binder (y vs. $n$ ) & 2.6029 & 0.8045 & 8.421 & 0.11029 \\
\hline Use of calcium channel blockers (y vs. $n$ ) & 1.6822 & 0.9516 & 2.974 & 0.07356 \\
\hline Model fit statistics (AIC-adding CAC) & \multicolumn{4}{|c|}{627.21 (final model with CAC) } \\
\hline loglink (without CAC) & \multicolumn{4}{|c|}{305.6} \\
\hline logling (with CAC) & \multicolumn{4}{|c|}{327.45} \\
\hline Comparison with vs. without CAC score & \multicolumn{4}{|c|}{ Chisq $43.697(p<0.001)$} \\
\hline
\end{tabular}

HR: Hazard Ratio; lower 0.95: lower boundary of the 95\% Confidence Interval; upper 0.95 upper boundary of the $95 \%$ Confidence Interval; ASCVD atherosclerotic cardiovascular disease defined if any of the following clinical data was reported: history of cerebrovascular disease; peripheral vascular disease; angina pectoris; history of myocardial infarction; aortic aneurysm; history of percutaneous coronary angioplasty with or without stenting; AIC: Akaike information criterion.

Furthermore, logistic regression models encompassing information regarding VC either in the coronary or aortic arteries allowed for a significant improvement in risk prediction (Table 3A-C). Indeed, addition of measurement of VC to the model, increased discrimination (C-statistics) and allowed a significant risk reclassification (IDI and NRI) of subjects new to hemodialysis $(p<0.001)$ (Table 3A-C). However, in this study cohort the logistic model that included the Agatston CAC score was not well calibrated (Calibration Chisquare; $p$ value $<0.001)$ and results may be less accurate that for other measurements of VC (Table 3A-C and Figure 2A-C). 
Table 3. Logistic regression was utilized to evaluate the incremental ability of each measurement of VC to predict all-cause mortality by computing the C-index, calibration statistic, integrated discrimination improvement (IDI) and continuous net reclassification improvement (NRI) of the most parsimonious model reported in Table 2A-C with or without measurement of VC. (A) Abdominal Aorta Calcification (AAC) evaluated via the Kauppila score; (B) Coronary Artery Calcification (CAC) evaluated via the Agatston score; $(\mathbf{C})$ Coronary Artery Calcification $(\mathrm{CAC})$ evaluated via the volume score.

\section{(A) Abdominal Aorta Calcification (AAC) Evaluated via the Kauppila Score}

Best Cutoff to Discriminate Expired vs. Alive Patients at Univariate Analyses 14.5 (specificity $67.0 \%$-sensitivity $78.3 \%$ )

\begin{tabular}{|c|c|c|c|}
\hline \multicolumn{4}{|l|}{ Metrics of discrimination-C-statistics ( $95 \% \mathrm{CI})$} \\
\hline without Kauppila & \multicolumn{3}{|c|}{$0.730(0.655-0.806)$} \\
\hline with Kauppila & \multicolumn{3}{|c|}{$0.841(0.782-0.900)$} \\
\hline Difference in C statistics (SD) & \multicolumn{3}{|c|}{$0.110(0.017) ; p$-value for comparison $<0.001$} \\
\hline \multicolumn{4}{|l|}{ Model fit statistics (AIC) } \\
\hline without Kauppila & \multicolumn{3}{|c|}{222.78} \\
\hline with Kauppila & \multicolumn{3}{|c|}{186.81} \\
\hline Comparison between models (ANOVA) & \multicolumn{3}{|c|}{ LR (Chisq) 37.9; d.f. $1(p<0.001)$} \\
\hline Metrics of calibration (adding Kauppila) & Chi-square & Df & $p$-value \\
\hline Hosmer-Lemeshow goodness of fit & 8.032 & 8 & 0.43 \\
\hline Patient reclassification (adding Kauppila) & Coeff & $95 \% \mathrm{CI}$ & $p$-value \\
\hline IDI $(95 \% \mathrm{CI})$ & 0.177 & $(0.123-0.232)$ & $p<0.001$ \\
\hline NRI categorical $(95 \% \mathrm{CI})$ & 0.246 & $(0.130-0.361)$ & $p<0.001$ \\
\hline NRI continuous $(95 \% \mathrm{CI})$ & 0.921 & $(0.623-1.220)$ & $p<0.001$ \\
\hline
\end{tabular}

(B) Coronary Artery Calcification (CAC) Evaluated via the Agatston Score

Best cutoff to Discriminate Expired vs. Alive Patients at Univariate Analyses 257.5 (specificity $80.9 \%$-sensitivity $73.9 \%$ )

Metrics of discrimination-C-statistics (95\%CI)

$\begin{array}{rc}\text { without CAC } & 0.742(0.669-0.8157) \\ \text { with CAC } & 0.901(0.854-0.947)\end{array}$

Difference in C statistics (SD)

0.158 (0.026); $p$-value for comparison $p<0.001$

Model fit statistics (AIC)

\begin{tabular}{rccc}
\hline without CAC & & 225.53 \\
\hline with CAC & & 160.18 \\
\hline Comparison between models (ANOVA) & & LR (Chisq) 67.3; d.f. 1 $(p<0.001)$ & $p$-value \\
\hline Metrics of calibration (adding CAC) & Chi-square & Df & 0.006 \\
\hline Hosmer-Lemeshow goodness of fit & 21.116 & 8 & $p$-value \\
\hline Patient reclassification (adding CAC) & Coeff & $95 \%$ CI & $p<0.001$ \\
\hline IDI (95\%CI) & 0.311 & $(0.241-0.381)$ & $p<0.001$ \\
\hline NRI categorical (95\%CI) & 0.301 & $(0.173-0.430)$ & $p<0.001$ \\
\hline
\end{tabular}


Table 3. Cont.

(C) Coronary Artery Calcification (CAC) Evaluated via the Volume Score

Best Cutoff to Discriminate Expired vs. Alive Patients at Univariate Analyses

66.5 (specificity $70.4 \%$-sensitivity $81.2 \%$ )

\begin{tabular}{|c|c|c|c|}
\hline \multicolumn{4}{|l|}{ Metrics of discrimination-C-statistics (95\%CI) } \\
\hline without CAC & \multicolumn{3}{|c|}{$0.776(0.707-0.845)$} \\
\hline with CAC & \multicolumn{3}{|c|}{$0.896(0.848-0.943)$} \\
\hline Difference in C statistics (SD) & \multicolumn{3}{|c|}{$0.120(0.021) ; p$-value for comparison $<0.001$} \\
\hline \multicolumn{4}{|l|}{ Model fit statistics (AIC) } \\
\hline without CAC & \multicolumn{3}{|c|}{214.03} \\
\hline with CAC & \multicolumn{3}{|c|}{162.83} \\
\hline Comparison between models (ANOVA) & \multicolumn{3}{|c|}{ LR (Chisq) 53.1; d.f. $1(p<0.001)$} \\
\hline Metrics of Calibration (adding CAC) & Chi-square & Df & $p$-value \\
\hline Hosmer-Lemeshow goodness of fit & 11.897 & 8 & 0.1558 \\
\hline Patient reclassification (adding CAC) & Coeff & $95 \% \mathrm{CI}$ & $p$-value \\
\hline IDI $(95 \% \mathrm{CI})$ & 0.241 & $(0.177-0.305)$ & $p<0.001$ \\
\hline NRI categorical $(95 \% \mathrm{CI})$ & 0.301 & $(0.173-0.429)$ & $p<0.001$ \\
\hline NRI continuous $(95 \% \mathrm{CI})$ & 1.072 & $(0.774-1.370)$ & $p<0.001$ \\
\hline
\end{tabular}

ASCVD: atherosclerotic cardiovascular disease defined if any of the following clinical data was reported: history of cerebrovascular disease; peripheral vascular disease; angina pectoris; history of myocardial infarction; aortic aneurysm; history of percutaneous coronary angioplasty with or without stenting; AIC: Akaike information criterion; IDI: integrated discrimination improvement; NRI: net reclassification improvement.

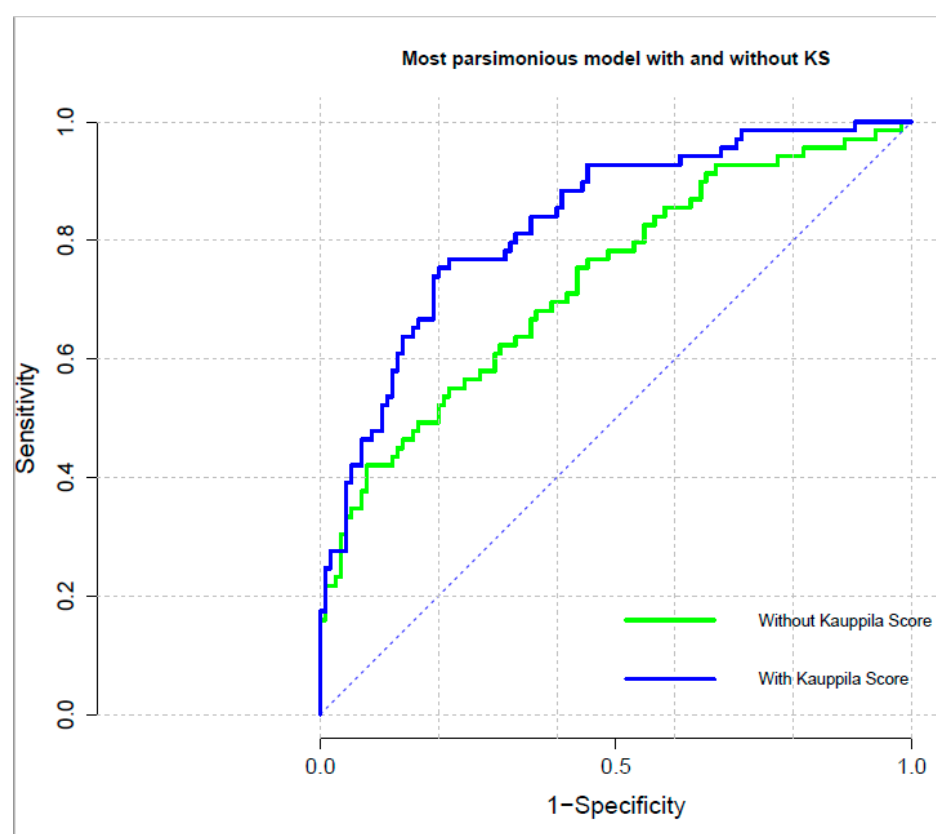

(A)

Figure 2. Cont. 


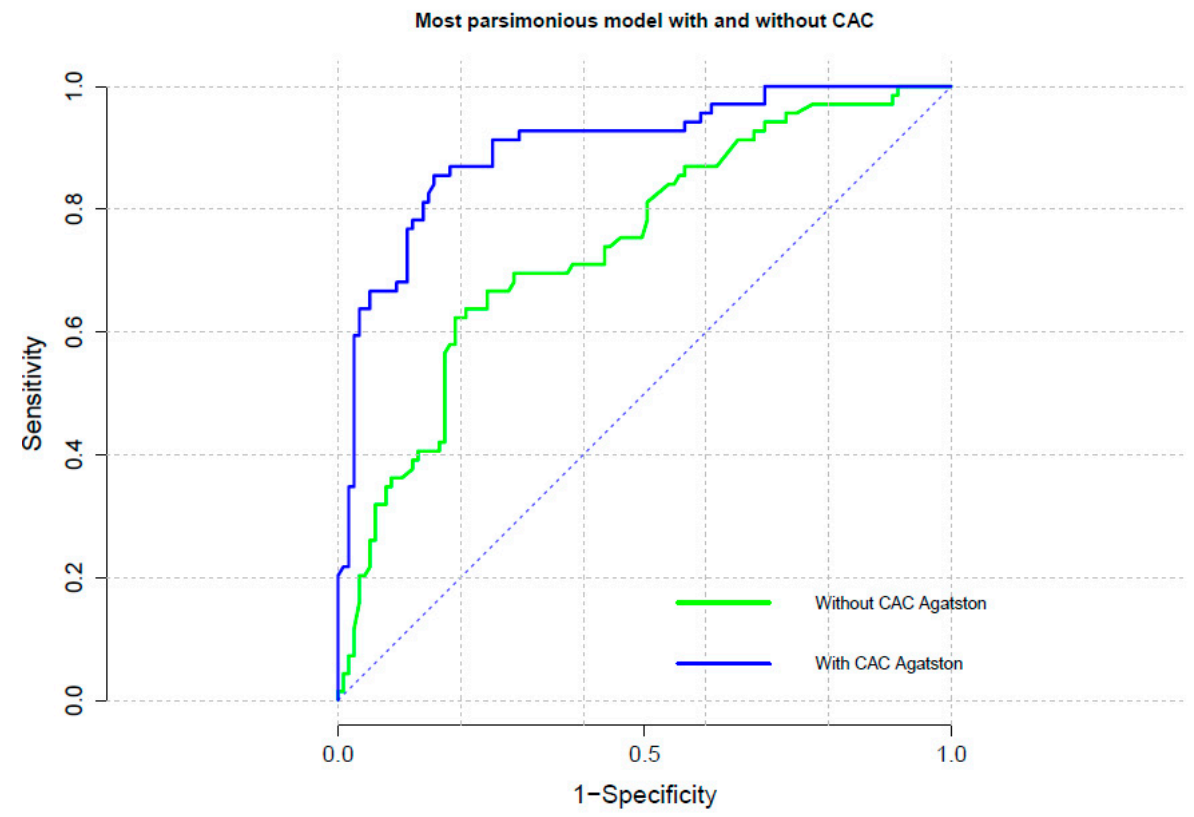

(B)

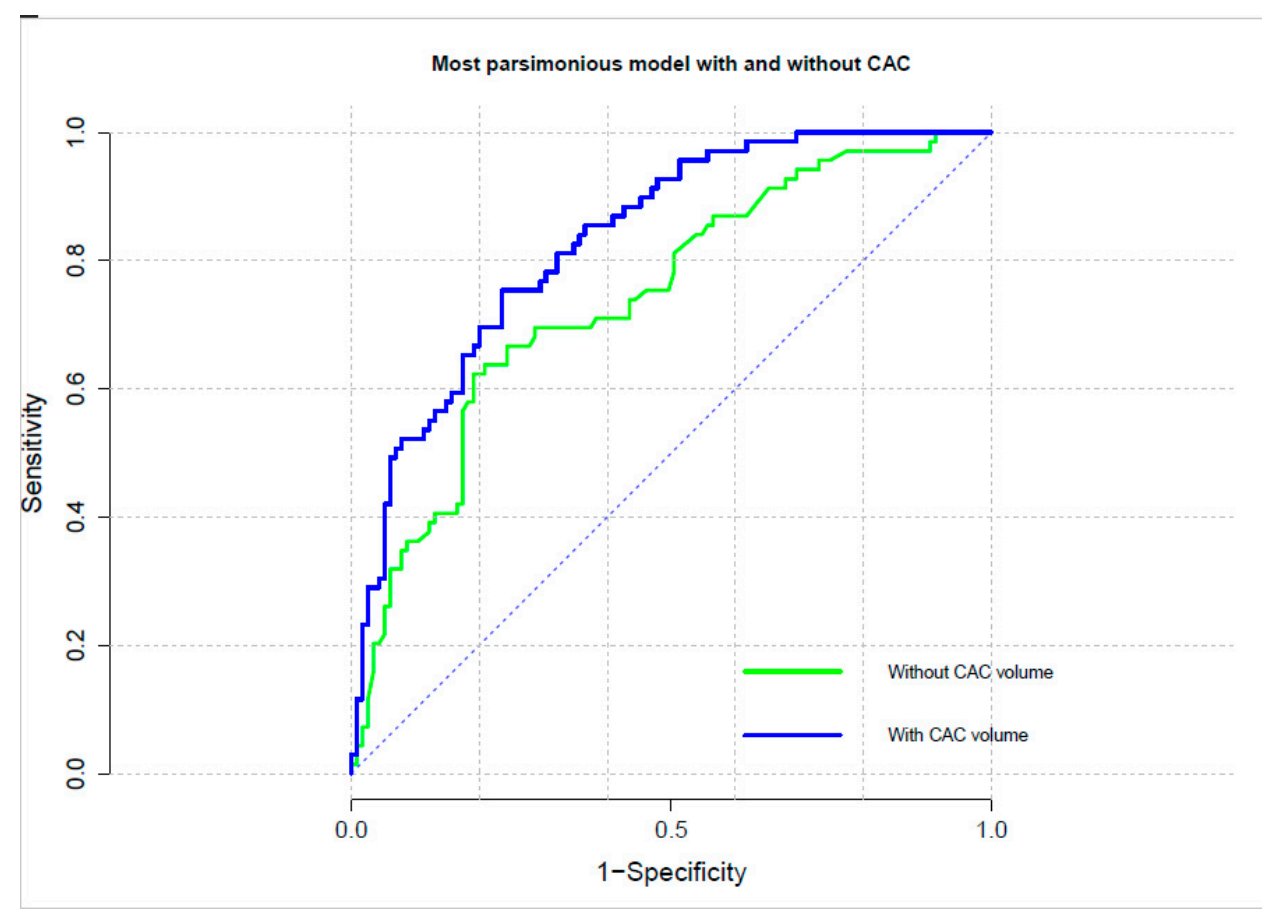

(C)

Figure 2. Receiver operating characteristic curves of most parsimonious models to predict all-cause mortality with and without measurement of VC. (A) Abdominal Aorta Calcification (AAC) evaluated by quartiles of Kauppila score (KS); (B) Coronary Artery Calcification (CAC) evaluated via the Agatston score; (C) CAC evaluated via the volume score.

\section{Discussion}

$\mathrm{VC}$ is a complex phenomenon involving active and passive mechanisms such as dysregulation of mineral metabolism [3,5,7,16-18] As renal function declines, prevalence of VC increases ranging from $40 \%$ in CKD stage 3 and 4 , to $60 \%$ in CKD stage 5 patients entering dialysis and $80-85 \%$ in prevalent hemodialysis patients $[5,7]$. Furthermore, data suggest a link of VC with poor survival and a variety of cardiovascular events such as acute and chronic 
coronary artery syndromes, heart failure, cardiac arrhythmias and sudden death $[5,7,18]$. Because CKD is also associated with excessive vascular risk, it is logical to assume that VC assessment may improve risk stratification and possibly personalized treatment(s) in subjects with impaired renal function. However, only few studies have investigated the predictive value of VC screening in different arterial sites (i.e., coronary arteries and abdominal aorta) in a cohort of patients starting dialysis.

Overall, the main findings of this study are that presence and extension of VC predicts risk of death in patients starting hemodialysis irrespective of the arterial site. Even though CAC quantitatively measures VC and is commonly perceived as a better predictor of outcome than abdominal aorta calcification, the difference is minimal and all measurements of VC allowed for a significant risk re-stratification beyond traditional risk factors for CV disease (i.e., Framingham risk score) or history of atherosclerotic vascular disease.

Current findings expand available evidence. Previous experience is limited to one method for VC assessment or have not evaluated the incremental predictive value of VC beyond traditional CV risk factors [5,18]. Furthermore, current results support the use of CAC or KS to identify high risk and fragile patients as suggested by the KDIGO clinical guidelines on CKD-MBD [7]. Indeed, a risk-based approach encompassing markers of vascular disease to guide management in patients receiving dialysis is advisable in light of the high vascular risk and poor predictive value of traditional $\mathrm{CV}$ risk factors in these subjects [17]. In these regards, CAC or KS are simple tools to assess the individual vascular risk.

Irrespective of the metabolic pathway or mechanism promoting VC, CAC and AAC are markers of tissue damage and their presence suggest a prolonged exposure to a vascular noxa that ultimately results in deposition of crystals of hydroxyapatite in the context of the arterial wall $[3,5,18]$. While circulating biomarkers reflect the instantaneous risk, tissue markers reflect the cumulative exposure to noxious factors to which an individual has been exposed [3]. In these regards, VC are less susceptible to transient perturbation of the subject's homeostasis and are stronger predictors of risk than circulating biomarkers.

Numerous techniques are available to measure VC $[1,5]$. Simple in office tools such as plain X-rays, 2D ultrasound and echocardiography or more sophisticated and such as electron beam computed tomography and multi-slice computed tomography can be used to detect and quantify VC in blood vessels as well as cardiac valves [1,5]. Quantification of VC with computed tomography (CT) is currently the gold standard to score VC. In this study, we compared the predictive value of two different CT scoring systems [8,9] for evaluating CAC and AAC assessed according to the methodology described by Kauppila et al [10]. While both the Agatston and the volume score quantitatively assess CAC [8,9], the KS semi-quantitatively (score range $0-24$ ) assess the abdominal aorta coursing in front of the lumbar spine [10]. However, CT exposes the patients to a greater radiation burden and is less available than plain X-ray used for KS [1].

Although the peculiar reasons for arterial vulnerability in CKD patients is not completely understood, several lines of evidence confirmed the association of CAC and KS and risk of death or CV events in different stages of CKD including patients on maintenance dialysis [5]. Overall, a graded increase in the risk of unfavorable prognosis is associated with progressively higher burden of calcification, irrespective of adjustments for confounders [5,15]. However, while previous observations have suggested a strong accordance among these measurements [1], no study has investigated whether these tools have different predictive value and which one should be used for risk prediction. Current results, suggest that independently of the arterial site investigated or the scoring system utilized, models encompassing VC significantly $(p<0.001)$ improve risk reclassification supporting the use in clinical practice of simple in-office tools such as plain X-ray for vascular calcification detection.

This piece of information may be of particular use in consideration of the potential therapeutic implication. Indeed, some lines of evidence suggest that CKD-MBD or anticoagulant management may impact the risk of VC deposition and progression as well as the 
risk of unfavorable outcomes [7,19-21]. Further, a recently published study showed that a newer compound SNF472 significantly attenuates CAC progression in hemodialysis patients [22], independently of baseline characteristics [23]. However, whether VC attenuation improves survival or reduces CV events await definitive confirmation in future studies.

This study has several limitations. The relatively small sample size and the retrospective nature are among the others the most relevant ones. Indeed, KS was not protocolized nor centralized and some differences between patients with and without this assessment in the Independent study cohort exist (Table S1). While these limit the generalizability of current findings, a rigorous method was used to control for potential confounders. Furthermore, the fact that all three measurements of VC yielded similar results suggest that the reported findings are consistent.

In conclusion, we documented in a cohort of patients new to dialysis that VC predict outcome and significantly improve risk stratification beyond traditional $\mathrm{CV}$ risk factors. Overall, it seems that CAC is a better predictor of outcome than abdominal aorta VC, though the difference is minimal. However, future efforts are needed to assess whether a risk-based approach encompassing VC screening to guide HD patient management improves survival.

Supplementary Materials: The following are available online at https:/ / www.mdpi.com/2077-038 3/10/3/376/s1. Table S1: comparisons between subjects enrolled in the Independent study with and without evaluation of abdominal aorta calcification (AAC) via the method described by Kauppila et al ${ }^{10}$; Table S2A: independent predictors of all-cause mortality in the study cohort 1; Table S2B: The most parsimonious model was then selected via a stepwise methods.

Author Contributions: Conceptualization, A.B., L.D.L. and B.R.D.I.; Data curation, B.R.D.I.; Formal analysis, A.B.; Writing-original draft, A.B.; Writing-review \& editing, L.D.L., D.R., R.C., M.M., C.L., C.R., M.F., M.C. and B.R.D.I. All authors have read and agreed to the published version of the manuscript.

Funding: This post hoc analysis of the Independent study received no external funding. All costs connected with the Independent study were covered by the Italian National Health Care System.

Institutional Review Board Statement: N/A for this post hoc analysis.

Informed Consent Statement: Informed consent was obtained from all subjects involved in the Independent study.

Data Availability Statement: The data of the Independent Study are not publicly available due to Data Protection restrictions.

Conflicts of Interest: Antonio Bellasi received speaking honoraria from Sanofi, Amgen and is a consultant for Sanifit. Maria Fusaro received honoraria from Amgen, Abiogen and Vifor Pharma. Mario Cozzolino received speaking honoraria from Baxter, Amgen, Vifor Pharma and research grant from Baxter. Domenico Russo, Roberto Ciarcia, Michele Magnocavallo, Carlo Lavalle, Carlo Ratti, Biagio Raffaele Di Iorio and Luca Di Lullo have nothing to disclose.

\section{References}

1. Bellasi, A.; Ferramosca, E.; Muntner, P.; Ratti, C.; Wildman, R.P.; A Block, G.; Raggi, P. Correlation of simple imaging tests and coronary artery calcium measured by computed tomography in hemodialysis patients. Kidney Int. 2006, 70, 1623-1628. [CrossRef] [PubMed]

2. Bellasi, A.; Raggi, P. Vascular imaging in chronic kidney disease. Curr. Opin. Nephrol. Hypertens. 2012, 21, 382-388. [CrossRef] [PubMed]

3. Bellasi, A.; Raggi, P. Vascular calcification in chronic kidney disease: Usefulness of a marker of vascular damage. J. Nephrol. 2011, 24, 11-15. [CrossRef] [PubMed]

4. Fusaro, M.; Cozzolino, M.; Plebani, M.; Iervasi, G.; Ketteler, M.; Gallieni, M.; Aghi, A.; Locatelli, F.; Cunningham, J.; Salam, S.; et al. Sevelamer Use, Vitamin K Levels, Vascular Calcifications, and Vertebral Fractures in Hemodialysis Patients: Results from the VIKI Study. J. Bone Miner. Res. 2020. [CrossRef] [PubMed]

5. Dilsizian, V.; Gewirtz, H.; Marwick, T.H.; Kwong, R.Y.; Raggi, P.; Al-Mallah, M.H.; Herzog, C.A. Cardiac Imaging for Coronary Heart Disease Risk Stratification in Chronic Kidney Disease. JACC Cardiovasc. Imaging 2020. [CrossRef] [PubMed] 
6. Mace, M.L.; Gravesen, E.; Nordholm, A.; Egstrand, S.; Morevati, M.; Nielsen, C.; Kjaer, A.; Behets, G.; D’Haese, P.; Olgaard, K.; et al. Chronic Kidney Disease-Induced Vascular Calcification Impairs Bone Metabolism. J. Bone Miner. Res. 2020. [CrossRef] [PubMed]

7. Ketteler, M.; Block, G.A.; Evenepoel, P.; Fukagawa, M.; Herzog, C.A.; McCann, L.; Moe, S.M.; Shroff, R.; Tonelli, M.A.; Toussaint, N.D.; et al. Executive summary of the 2017 KDIGO Chronic Kidney Disease-Mineral and Bone Disorder (CKD-MBD) Guideline Update: What's changed and why it matters. Kidney Int. 2017, 92, 26-36. [CrossRef] [PubMed]

8. Agatston, A.S.; Janowitz, W.R.; Hildner, F.J.; Zusmer, N.R.; Viamonte, M.; Detrano, R. Quantification of coronary artery calcium using ultrafast computed tomography. J. Am. Coll. Cardiol. 1990, 15, 827-832. [CrossRef]

9. Callister, T.Q.; Cooil, B.; Raya, S.P.; Lippolis, N.J.; Russo, D.J.; Raggi, P. Coronary artery disease: Improved reproducibility of calcium scoring with an electron-beam CT volumetric method. Radiol. 1998, 208, 807-814. [CrossRef]

10. Kauppila, L.I.; Polak, J.F.; Cupples, L.A.; Hannan, M.T.; Kiel, D.P.; Wilson, P.W. New indices to classify location, severity and progression of calcific lesions in the abdominal aorta: A 25-year follow-up study. Atherosclerosis 1997, 132, 245-250. [CrossRef]

11. Di Iorio, B.; Molony, D.; Bell, C.; Cucciniello, E.; Bellizzi, V.; Russo, D. Sevelamer versus calcium carbonate in incident hemodialysis patients: Results of an open-label 24-month randomized clinical trial. Am. J. Kidney Dis. 2013, 62, 771-778. [CrossRef] [PubMed]

12. Di Iorio, B.R.; Cucciniello, E.; Bellizzi, V. Vascular calcification and QT interval in incident hemodialysis patients. J. Nephrol. 2009, 22, 694-698. [PubMed]

13. Wilson, P.W.; D’Agostino, R.B.; Levy, D.; Belanger, A.M.; Silbershatz, H.; Kannel, W.B. Prediction of Coronary Heart Disease Using Risk Factor Categories. Circulation 1998, 97, 1837-1847. [CrossRef] [PubMed]

14. Raggi, P.; Bellasi, A.; Ferramosca, E.; Block, G.A.; Muntner, P. Pulse Wave Velocity Is Inversely Related to Vertebral Bone Density in Hemodialysis Patients. Hypertensionaha 2007, 49, 1278-1284. [CrossRef] [PubMed]

15. Block, G.A.; Raggi, P.; Bellasi, A.; Kooienga, L.; Spiegel, D. Mortality effect of coronary calcification and phosphate binder choice in incident hemodialysis patients. Kidney Int. 2007, 71, 438-441. [CrossRef]

16. Bellasi, A.; Di Lullo, L.; Raggi, P. Cardiovascular calcification: The emerging role of micronutrients. Atherosclerosis 2018, 273, 119-121. [CrossRef]

17. Raggi, P.; Giachelli, C.; Bellasi, A. Interaction of vascular and bone disease in patients with normal renal function and patients undergoing dialysis. Nat. Clin. Pr. Neurol. 2007, 4, 26-33. [CrossRef]

18. Nelson, A.J.; Raggi, P.; Wolf, M.; Gold, A.M.; Chertow, G.M.; Roe, M.T. Targeting Vascular Calcification in Chronic Kidney Disease. JACC Basic Transl. Sci. 2020, 5, 398-412. [CrossRef]

19. Jamal, S.A.; VanderMeer, B.; Raggi, P.; Mendelssohn, D.C.; Chatterley, T.; Dorgan, M.; E Lok, C.; Fitchett, D.; Tsuyuki, R.T. Effect of calcium-based versus non-calcium-based phosphate binders on mortality in patients with chronic kidney disease: An updated systematic review and meta-analysis. Lancet 2013, 382, 1268-1277. [CrossRef]

20. Di Lullo, L.; Tripepi, G.; Ronco, C.; D’Arrigo, G.; Barbera, V.; Russo, D.; Di Iorio, B.R.; Uguccioni, M.; Paoletti, E.; Ravera, M.; et al. Cardiac valve calcification and use of anticoagulants: Preliminary observation of a potentially modifiable risk factor. Int. J. Cardiol. 2019, 278, 243-249. [CrossRef]

21. Di Lullo, L.; Tripepi, G.; Ronco, C.; De Pascalis, A.; Barbera, V.; Granata, A.; Bellasi, A. Safety and effectiveness of rivaroxaban and warfarin in moderate-to-advanced CKD: Real world data. J. Nephrol. 2018, 31, 751-756. [CrossRef] [PubMed]

22. Raggi, P.; Bellasi, A.; Bushinsky, D.; Bover, J.; Rodriguez, M.; Ketteler, M.; Sinha, S.; Salcedo, C.; Gillotti, K.; Padgett, C.; et al. Slowing Progression of Cardiovascular Calcification With SNF472 in Patients on Hemodialysis: Results of a Randomized Phase 2b Study. Circulation 2020, 141, 728-739. [CrossRef] [PubMed]

23. Raggi, P.; Bellasi, A.; Sinha, S.; Bover, J.; Rodriguez, M.; Ketteler, M.; Bushinsky, D.A.; Garg, R.; Perelló, J.; Gold, A.; et al. Effects of SNF472, a Novel Inhibitor of Hydroxyapatite Crystallization in Patients Receiving Hemodialysis-Subgroup Analyses of the CALIPSO Trial. Kidney Int. Rep. 2020, 5, 2178-2182. [CrossRef] [PubMed] 